\title{
SUMMARY REPORT OF NEUTRAL DETECTOR SUBGROUP *
}

A. Hadron Calorimeters at ISAbelle

Several aspects of calorimeter design and performance have been investigated by this subgroup. The primary focus of our consideration has been an evaluation of techniques, currently available or under development, which have possible applications at Isabelle. Thus, much of this report is taken from published information listed in the Bibliography.

By now, considerable experience has been gathered ${ }^{1}$ on the performance of shower detectors and calorimeters. Sophisticated computer programs exist ${ }^{2}$ which simulate these devices and can predict the behavior of new devices. The devices which we consider are generically the same: they all contain enough material. to contain a hadron cascade. They have a series of dense metal plates $(\mathrm{Fe}, \mathrm{Pb}, \mathrm{U} \ldots)$ to initiate the cascade; interspersed are active detectors which sample the ionization of particles transversing them (scintillator, liquid argon ion chambers, proportional wire chambers). There are important physical limitations on the energy resolution in calorimeters ${ }^{3,4}$ which influence the design of a specific device.

Design of calorimeters for specific experiments at Isabelle depends critically on the physics goal. A separate report

* C. Akerlof, J. Bensinger, G. Donaldson, R.M. Edelstein,

W. Friskin, M. Goldberg, H, Gordon, P. Grannis, N, Horowitz,

S. Ozaki, I. Pless, D. Rahm, P. Rehak, I. Rosenson, C.D. Wheeler.

1. M. Atac, Editor, Proceedings of the Calorimeter Workshop at Fermi National Laboratory, May 1975.

2. T, A, Gabriel and W. Schmidt, Nucl. Instr, and Meth. 134, 271 (1976).

3. C, W. Fabjan, W. Struczinski, W.F. Willis, C. Kourkoumelis, A. J. Lankford and P. Rehak, Nucl. Instr, and Methods 141, 61 (1977).

4. C. W. Fabjan and W.J. Willis, Ref. 1, Page I, 


\section{DISCLAIMER}

This report was prepared as an account of work sponsored by an agency of the United States Government. Neither the United States Government nor any agency Thereof, nor any of their employees, makes any warranty, express or implied, or assumes any legal liability or responsibility for the accuracy, completeness, or usefulness of any information, apparatus, product, or process disclosed, or represents that its use would not infringe privately owned rights. Reference herein to any specific commercial product, process, or service by trade name, trademark, manufacturer, or otherwise does not necessarily constitute or imply its endorsement, recommendation, or favoring by the United States Government or any agency thereof. The views and opinions of authors expressed herein do not necessarily state or reflect those of the United States Government or any agency thereof. 


\section{DISCLAIMER}

Portions of this document may be illegible in electronic image products. Images are produced from the best available original document. 
investigates the requirements imposed by several specific experiments. However we note here that experiments requiring a) good hadron to electron discrimination, b) coarse energy resolution over large solid angle or c) good resolution in limited solid angle, dictate different design strategies. specific environmental factors which must be considered are:

a) Rate. At a luminosity of $=2 \times 10^{32} / \mathrm{cm}^{2} / \mathrm{sec}$ the event rate is $\sim 10$ MHz and the particle rate is $\sim 200 \mathrm{MHz}$. For a forward calorimeter at $25 \mathrm{mrad}$ with $100 \mathrm{~cm}^{2}$ cells placed $5 \mathrm{~m}$ from the interaction point there would be $\sim 10^{6}$ particles/sec in the cell. 5

Although it is possible to adjust the flux of particles incident on a unit of detector by positioning small angle detectors at large distances from the crossing region - there is clearly a premium on detectors with short resolving time. conventional PWC and wide gap liquid argon cells will have trouble at full luminosity.

b) Energy Resolution. Energy resolution is determined by many effects. Some of the factors involved are leakage of neutrinos, neutrons and muons through the boundaries of the detector; fluctuations due to sampling or, for a fine grained sampling device, fluctuations in the nuclear breakup process, and limitations of the ionization detector such as saturation and noise. 5. Frank E, Paige, "Charged Particle Production at IsAbelle". submitted to this workshop. 
For the best resolution, showers must be essentially contained, which at $10 \mathrm{GeV}$ requires $\sim 5$ absorption lengths. This grows only logarithmicaliy with energy to 6 or 7 absorption lengths at $400 \mathrm{GeV}$. An indication of the effect of sampling frequency on resolution is given by the. following "rule of thumb". The rms width of the energy response due to sampling is given by $\sigma \dot{t} c \sqrt{E \cdot t}$, where $E$ is the energy of the shower, $t$ is the sampling thickness $\left(\mathrm{g} / \mathrm{cm}^{2}\right.$ of one plate and one sampling layer), C is a constant which depends only on the shower type (electromagnetic or hadronic) and the composition of the calorimeter. For example, $c \approx .06:\left(\mathrm{GeV} / \mathrm{g} / \mathrm{cm}^{2}\right)^{1 / 2}$ for lead-filled electromagnetic calorimeters, ${ }^{6}$ and $c \dot{\sim} .15\left(\mathrm{GeV} / \mathrm{g} / \mathrm{cm}^{2}\right)^{1 / 2}$ for iron-filled hadron calorimeters. ${ }^{2}$ Finally for hadron showers resolution can be improved substantially by compensating for the energy lost due to nuclear binding energy by using uranium in place of iron. ${ }^{3}$

c) Service. Since access to equipment is limited, any device. at the ISA will have to be "hardened". This is a particular concern in devices with a large number of PWC's or phototubes, such as calorimeters. Further, the calorimeters should be designed in a modular way to permit easy servicing. specific precautions against "spikes" in instantaneous rate (accidental beam losses, etc.) must be taken. In addition we are concerned about the extent to which the detectors can withstand radiation damage.

6. D, Hitlin, J.F. Martin, C.C. Morehouse, G.S. Abrams, D. Briggs, W. Carithers, S. Cooper, R. Devoe, C, Friedberg, D. Marsh, S. Shannon, E. Vella, and J.S. Whitaker, Nuc. Instr, and Meth. 137, 225 (1976). 
d) Segmentation. Many applications of calorimetry demand some position information on the incident particles. Uniformity of response and rate also set limits on module transverse dimensions. We envision individual detectors of transverse dimensions of order $100-400^{\circ} \mathrm{cm}^{2}$. This size is also dictated by the transverse shower dimensions. $2,3,6$ Longitudinal segmentation is a useful feature as it provides additional information which can aid offline energy resolution and electron/hadron discrimination. The front end of the calorimeter should use $\mathrm{Pb}$ plates for electromagnetic identification, followed by iron or Uranium plates for hadron showers. The need for, segmentation and for close packing of individual modules impose limitations upon the means of ex-. tracting ionization information from a module to the outside. Electronic signals are more convenient than light guides, although the use of fluorescent wave shifters can allow the close packing of scintillator elements.

e) Calibration. The above considerations suggest many detectors will be employed in a calorimeter: Each must be calibrated and monitored to maintain an absolute energy measurement capacity. Such calibration schemes are cumbersome for scintillator-phototube arrays with several thousand elements. They are relatively simpler with the electronic readouts of ionization chambers.

\section{f) Readout Electronics. The large number of cells required} suggest that the recording of analog signals could become expensive and cumbersome if conventional ADC systems are used. Attention should be given to schemes which can suppress zero-signal 
channels and which serialize the analog information to a single ADC. Promising developments on CCD's (charge coupled devices) are discussed elsewhere in the proceedings of this workshop. 7 g) Shower Type. Many of the design problems of electromagnetic calorimeters are similar to those of hadron calorimeters: In this report we will discuss primarily hadron calorimeters, however, in many cases similar considerations apply to electromagnetic shower detectors.

Two basic choices exist in designing a calorimeter for measuring hadron energies: the shower-producing plate material (Fe vs U) and the ionizationidetector (PWC vs scintillator vs liquid argon). These choices are not totally decoupled, for example, the use of PWC's in a uranium environment appeared to. us to be unfeasible due to the natural radioactive background.

The basic distinction between Fe and. U calorimeters is simplicity and economy vs resolution. The ultimate resolution for iron plate calorimeters is determined by fluctuations in the amount of energy consumed in disrupting iron nuclei during the hadronic cascade. This depends on the number and energy of $\pi^{\circ}$ 's produced in the early interactions in the cascade which then determines the fraction of energy in the hadronic component (vs. the electromagnetic component) of the shower. A thin plate iron calorimeter gives approximately $30 \%$ less response to hadrons than to electrons at $10 \mathrm{GeV}$. In addition, the hadronic shower spectrum is skewed toward the high energy side due to "charge exchange 7. Report by Mike zelier etal., submitted to this workshop. 
type" events. This high energy tail makes unfolding the momentum spectrum of the primary particle difficult.

Iron plate calorimeters are, however, relatively simple to design and construct, and are relatively cheap. The shower can be sampled adequately by plastic scintiliator, so there. will be many applications where speed of response, ease of service and construction, absence of awkward peripherals (cryogenic tank, e.g.l will outweigh the desire for ultimate resolution. It should be noted that there may also emerge some as yet unexploited technique for compensating for the binding energy losses on an event by event basis, perhaps by detecting the neutrons associated with'nuclear interactions separately, and appropriately mixing the signals. Until such time, iron plate calorimeters will remain convenient devices of rather coarse energy resolution.

Uranium plate devices are attractive when energy resolution is at a premium. As a specific exercise, we have set ourselves the problem of evaluating a uranium-scintillator detector and comparing its capability with that of a uranium-liquid argon device which has been built. ${ }^{3}$ our motivation for discussing scintillator elements is their superior time resolution and consequent higher rate capability. We outline first the mechanism for recovery of binding energy losses in uranium so as to apply the process to scintillator calorimeters. 
Collisions of hadrons which involve breakup of nuclei give, on average, less visible ionization energy than those collisions leading to a large fraction of energy in electromagnetic showers. These breakup collisions also give rise to a fairly large number of neutrons. In uranium, these neutrons can be used to inace fissions with consequent release of energy. Thus the energy deposited in the calorimeter can be compensated - on an event by event basis - so that hadron-electron cascades release equal visible ionization. By equalizing ionization produced by electromagnetic and hadronic components of the shower the resolution is reduced by a factor of approximately two över iron plate calorimeters. It should be noted that the uranium calorimeter that has been tested ${ }^{3}$ was of 1 imited transverse dimensions $\sim 25 \mathrm{~cm}$, . perhaps when a full scale calorimeter will be constructed of all uranium the binding energy may be over-compensated.

The energy recovered from fission comes primarily from gamma rays emitted in the $1 \mathrm{MeV}$ range. In the case of liquid Argon as the ionization detector, with few hundred nanosecond collection times, the gamma energy is released fast enough to be in coincidence with the ionization produced by cascade particles. In contemplating a U-scintillator calorimeter, the time resolution of $\approx 20$ nsec implies tighter requirements on the time of gamma/ energy releast.

The sequence of events leading to decay gamma's is:

1. Primary collisions of hadron and nucleons with prompt neutrons evaporation. 
2. "Diffusion" of these neutrons to ${ }^{238}$ u nucleus where a fission occurs. The fission cross section is $\sigma \approx 0.5 \mathrm{~b}$ for $2 \leq E_{n} \leq 7 \mathrm{MeV}$ and somewhat larger for $E_{n}>7 \mathrm{MeV}$. Thus'the mean free path for fission is $\# 40 \mathrm{~cm}$ in solid $U$. (The interaction mean free path is about 6 times shorter.)

3. In the fission reaction, several neutrons are evaporated within $10^{-13}$ sec (before separation of the fission daughter nucleil. 8 The daughter nuclei then execute chains of decays, yielding at several steps $\gamma^{\prime} s$ of $\sim 1$ MeV each. These fission daughters are highly excited and in high angular momentum states so the chains may involve mary steps - some with long (usec) decay times. However, there is reasonable evidence that a substantial fraction of $\gamma$ energy is released within a few nsec. 9 The energy of the de-excitation $\gamma^{\prime}$ s falls exponentially with $\gamma$-ray energy with a mean value around $1 \mathrm{MeV}$, there are about 8 such $\gamma^{\prime} s$ per fission. The $\gamma$ spectra are essentially independent of neutron energy or particular final state fission products. 4. In addition to fission, the few MeV neutrons can induce nuclear reactions. which release $\gamma^{\prime} s$ - in particular $n+238_{U}+$ $n^{\prime}+238 U^{*}$. The decay of $238 U^{*}$ yields $\gamma^{\prime}$ s of energy $\sim 1 \mathrm{MeV}$ which add to the regained energy in the cascade. ${ }^{10}$ These $\gamma^{\prime} s$ are emitted in times less than a few nanoseconds:

8. J.S. Fraser, Phys, Rev, 88, 536 (1952).

9. T.A. Gabriel, ORNL/TM-57 $\overline{69}$; Y.S. Zamyatin in Physics of Nuclear Physics, ed. N, Perfilov and V.P. Eismont (Israel Program for Scientific Translations 1964).

10. East Neutron Physics, ed. J.B. Marion and J.I. Fowler, Ch, V.I and V.K, Interscience Publishers (New York). 
5. Finally, for neutron energies above $\sim 10 \mathrm{MeV}$, inelastic reactions in which two neutrons are emitted become possible.

With the above information, we can estimate the time over which most of the $\gamma$ energy is released. This is determined by the time lapse associated with the neutron time-of-flight from the initial collision to the point where some $\gamma$ emitting nucleus is formed. Given the mixture of fission and inelastic collisions ${ }^{3}$ and the mean density in typical uranium calorimeters, this time lapse is estimated to be about 5 nanoseconds. Fission $Y^{\prime} s$ appear within a few nanoseconds of neutron arrival and excited $238 \mathrm{U}$ decay is essentially prompt. It thus appears that the $Y$ energy is present in the shower in usable form ( 1 MeV per $\gamma$ ) within 10 nanoseconds: of the primary shower particles and that scintillator response times will include most ( 7 . 75 ) of the total available energy release from fission associated events. An experimental test of this conclusion is essential. Better information on the neutron flight times should be acquired. A requirement on the calorimeter construction is imposed by the energy of $y$-rays. Uranium plates should be kept thin enough that there is not appreciable $\gamma$ absorption.

Containment of a hadronic shower requires about 5 absorption lengths at $10 \mathrm{GeV}$ and increases to $6-1 / 2$ absorption lengths at $400 \mathrm{GeV}$. The absorption length in $\mathrm{U}^{\circ}$ is about $10 \mathrm{~cm}$, so many sampling planes ( $\geq 100)$ are necessary in order to realize the potential gain from energy compensation by fission. 
A final problem is the radioactive background induced in the uranium and sensed continually by the active detector. A measurement ${ }^{I 1}$ has been performed of the radioactivity induced in ${ }^{238} \mathrm{U}$ by incident protons of $740 \mathrm{MeV}$. An. incident flux of $3 \times 10^{.11}$ protons/sec yields an equilibrium activity, recorded $40 \mathrm{~cm}$ from the sample, of $10 \mathrm{R} / \mathrm{hr}$ which decays to $1 \mathrm{R} / \mathrm{hr}$ in $3 \mathrm{hr}$. Inferring a dominant decay time of $1.3 \mathrm{hr}$, using the conversion $1 \mathrm{R}=3360$ erg/cm of $Y$ flux $\left(0.06 \leq E_{Y} \leq 2 \mathrm{MeV}\right)$, and assuming a mean energy release of $1 \mathrm{MeV}$ per radioactive decay, we can establish that the number of disintegrations per sec in uranium is approximately

$$
\text { dN/dt } 20.4 E_{0} \text { (incident hadron rate), }
$$

where $\Sigma_{0}$ is the hadron energy in GeV. Thus we expect, for a calorimeter at. ISABELLE where $E_{0} *$ Flux $\approx 3 \times 10^{8} \mathrm{GeV} / \mathrm{sec}$ is incident, about $10^{8}$ disintegrations per second. It. would seem that reasonable segmentation of the active detector, both in the longitudinal and transverse directions, should reduce this rate to manageable proportions. However, the background radiation is a worry for scintillator (or PWC) calorimeters and these ideas should be tested.

At this point we must interject some notes of caution about uranium. Even when the uranium is free and comes in a metallic form, the cost of casting, rolling and shearing is approximately 2 to 3 dollars per pound, about 10 times the cost of iron. Uranium oxide is a black powder which can flake off easily. If this presents a problem, plating the uranium can add up to a I1. L.R. Veeser, R.R. Fullwood, A.A. Robba and E.R. Shunk, Nucl. Instr, and Meth. 117, 509 (1974). 
dollar a pound. Operations on uranium such as shearing and punching holes are quite sage, however, machining presents special problems. Uranium is pyrophoric (dust will ignite spontaneously when exposed to air) and special care must be taken to prevent fires.. In addition since uranium is radioactive (although mildly), Federal and state regulations ${ }^{12}$ must be obeyed while shipping, which of course increases the cost. We have only considered using acrylic scintillator with wave shifter bars for light collection. The acrylic scintillator has relatively long attenuation length, low light output, low cost, the surface does not deteriorate as quickly as polystyrene and it is easy to work with. For a canonical uranium calorimeter of $2101.5 \mathrm{~mm}$ sheets one would use $3 \mathrm{~mm}$ sheets of scintillator. They are available from Rohm in Germany for $\because \$ 50 / \mathrm{sq}, \mathrm{m}$, or $\sim \$ 10 \mathrm{~K} / \mathrm{sq}, \mathrm{m}$, for the entire depth of the calorimeter. The number of phototubes for a $64 \mathrm{~m}^{2}$ calorimeter with $20 \times 20 \mathrm{~cm}$ modules is $\sim 1600$. The effect of using wave-shifter strips in the radioactive environment of uranium has not been considered. This too should be examined in a test program.

As noted above PWC's will not work in a uranium calorimeter because of high background radioactivity. In addition for a fine grained calorimeter with plates 1.5 to $3 \mathrm{~mm}$ thick, PWC ionization collectors $1 \mathrm{~cm}$ thick would make the calorimeter prohibitively large. Therefore, if hadron calorimeters require 12. Title 49. Code of Federal Regulations, Part 173.389. 
uranium to achieve satisfactory energy resolution then PWC's are ruled out as detectors. However, if PWC's prove to be significantly cheaper or easier to use, one might consider using them in the "front end" as an electromagnetic calorimeter consisting of PWC-Pb cells, We considered, in this connection, whether. PWC's could compete with scintillators regarding energy resolution. Following Katsura et al. 13 one can assume the energy resolution has two components: one due to fluctuations in $N$, the number of gap crossings, the other due to fluctuations in charge (or light) produced during those gap crossings. For 1.0 (10.0) GeV showers the resolution due to fluctuations in $N$ is expected to be approximately .19 (.06) FWHM, the resolution due to fluctuations in the charge collected (assuming uniform gas gain). .18 (.17) \& FWM and the resolution due to light collected (assuming uniform light collection efficiency) .13 (.12) \& FWHM. The estimated energy resolution due to fluctuations in the charge produced during gap crossings was taken from Aderholz 14 for the $1 \mathrm{GeV}$ case and from Landau theory for the $10 \mathrm{GeV}$ case. No account was taken of possible effects of $x$-rays in the KeV range accompanying the shower. If the above numbers tell the whole story then PWC's are almost as good as scintillators as far as energy resolution is concerned.

13. T. Katsura, S. Parker, V.Z. Peterson, D.E. Yount and M.L. Stevenson, Nucl. Instr, and Meth. 105, 245 (1972).

14. M. Aderholz, P. Lazeyras, I. Lehraus, R. Matthewson and W. Tejessy, Nucl. Instr. and Meth, 118, 419. (1974). 
Some years ago Nordberg (see Ref. 13) tested a prototype PWC calorimeter. At that time, the observed resolution at 1,0 $(8,0) \mathrm{GeV}$ was about .50 (.20) \% FWM,

As a result of these considerations we feel the uraniumScintillator Calorimeter holds substantial promise for use at ISA. There are however a substantial number of potential problems and open questions, some of which have been mentioned in this report, We urge Brookhaven to start a substantive test program toward developing detectors of this type.

B. Liquid Argon Calorimeters.

We will discuss some advantages and disadvantages of liquid Argon (L Ar) sampling calorimeters including some of the experience gained in running at the CERN Intersecting storage Rings. The use of liquid Ar in an ion chamber to sample the ionization developed in heavy metal plates and in the Ar was initiated by W.J. Willis and developed as a useful technique by willis and co-workers. is-17

\section{Advantages}

With a I Ar calorimeter one can use thin absorber plates because of the ease of collecting the charge and of calibrating the system. This leads to small sampling fluctuations which is often the limiting factor in the energy resolution of a calorimeter.

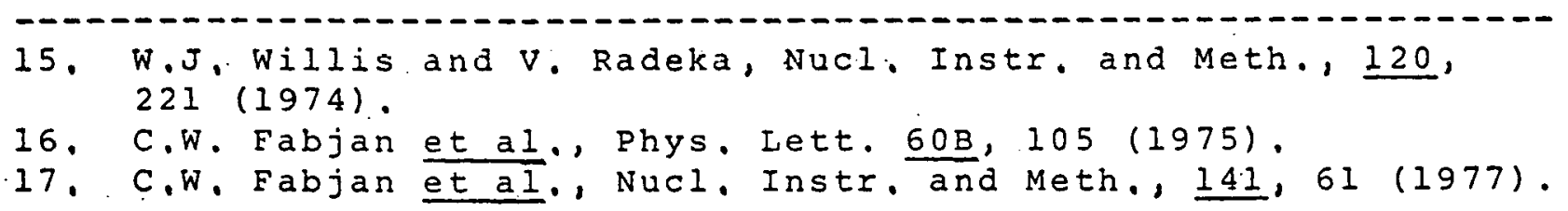


The calorimeter can be subdivided into narrow strips to determine the position of the particle shower. For the case of electromagnetic showers and using lead plates, the subdivision can profitably be as small as $1 \mathrm{~cm}$. Each section of the calorimeter can be calibrated in an absolute fashion using a precision pulser and stable capacitors. Further calibrations can be obtained by using straight through muons from cosmic rays. The resolution for electromagnetic showers can reach a standard deviation $\sigma / E=0.04 / \sqrt{E}$ (with $E$ in GeV). For hadron showers, using uranium plates to compensate for nuclear binding effects, the resolution can reach $\sigma / E:=0.32 / \sqrt{E}$. I Ar calorimeters can be constructed to cover large solid angles.

\section{Disadvantages}

The time response of a $L$ Ar ion chamber is basically limited by the drift time of the electrons in the liquid Ar. The duration of the shaped pulse for practical devices has been of the order of 1 micro sec and pileup problems can result for rates per strip comparable to this figure. The time resolution possible in the $L$ Ar is, however, \pm 10 nsec and it would be possible (not easy or cheap) to design the electronics to reject events with pileup and to pick out the correct event from several events occurring within 1 usec. Unfortunately the precise time information is only available rather late, which complicates using the calorimeter in the trigger.

By speeding up the electron collection time by adding methane to the $L$ Ar and changing the pulse shaping in the electronics it 
should be possible to get down to total pulse durations of 200 nsec, but at the expense of somewhat higher noise.

A further disadvantage is the necessity of operating in a cryogenic environment with all the attendant expense and operating problems. The necessity for thernal insulation and cryogenic supports complicates the installation of I Ar calorimeters and the full coverage of the desired solid angle.

\section{Electromagnetic Shower Detectors}

For electron and gamma ray detectors, lead is a natural choice for the heavy metal. It is possible to construct an 18 radiation length detector as short as $30 \mathrm{~cm}$ in depth and with an energy. resolution $\sigma / E=0.10 / \sqrt{E}$. For the best obtainable resolution one needs $60 \mathrm{~cm}$ depth with lead plates $0.5 \mathrm{~mm}$ thick and I Ar gaps of $2 \mathrm{~mm}$. This will give $\sigma / \mathrm{E}=0.04 / \sqrt{\mathrm{E}}$. All attempts so far to obtain a better resolution using pb plates have not succeieded.

The two obvious choices for geometrical subdivision of the calorimeter are into strips and into small rectangular stacks that we call "towers". The strips can be made as narrow as one $\mathrm{cm}$ and must run in several directions so as to permit geometrical reconstruction. We found from experience at the ISR that the several directions should be interleaved in depth so that the similar pulse heights can be used as an additional constraint for the geometrical construction. The stacks should also be subdivided in shower depth so as to permit hadron rejection. A hadron rejection of a factor of 100 can be achieved. 


\section{Hadron Calorimeters}

The best resolution should be obtained with thin (1.3 $\mathrm{mml}$ plates of depleted $U$ with a $1.3 \mathrm{~mm} L$ Ar gap. The fisision of the U produces sufficient additional ionization so that the most probable signal will be the same for electromagnetic showers and for hadron showers. Radioactivity from the U produces small pileup pulses which somewhat increases the noise. Since the natural width of a hadron shower is about $15 \mathrm{~cm}$, subdivision into towers of about this size is feasible. The ambiguity problem is less, but at the expense of decreased resolution.

5. Combined Calorimeter.

A calorimeter suitable for both electromagnetic and hadronic events could be constructed by making the front section.. of narrow $\mathrm{Pb}$ strips to start and develop the electromagnetic shower followed by a section of $U$ towers to contain hadronic showers. The lead in the front would shield the rest of the apparatus from U radioactivity. The Pb strips would be divided into 2 depths and 3 directions. A calorimeter of this type would be able to measure the energy and position of all particles, except neutrinos and muons, coming from an interaction.

Research carried out, in part, under the auspices of the United States Department of Energy under Contract No. EY-C-02-0016. 